\title{
Limited Specificity in the Injury and Infection Priming against Bacteria in Aedes aegypti Mosquitoes
}

\author{
Valeria Vargas ${ }^{1,2}$, Miguel Moreno-García ${ }^{1,3 *}$, Erika Duarte-Elguea ${ }^{1}$ and \\ Humberto Lanz-Mendoza ${ }^{\text {* }}$ \\ ${ }^{1}$ Centro de Investigaciones Sobre Enfermedades Infecciosas, Instituto Nacional de Salud Pública, Cuernavaca, Mexico, \\ ${ }^{2}$ Posgrado de Ciencias Biológicas, Universidad Nacional Autónoma de México, Mexico City, Mexico, ${ }^{3}$ Department of \\ Microbiology, Immunology and Pathology, Colorado State University, Fort Collins, CO, USA
}

Injury and infection priming has been observed in several insect groups, reported as host immune protection against contact with a pathogen caused by a previous infection with the same. However, the specific response against a pathogen has not been demonstrated in all insect species. Investigating the specific priming response in insects is important because their immune strategies probably reflect particular selective pressures exerted by different pathogens. Here, we determined whether

OPEN ACCESS

Edited by: Heinrich Korner, Menzies Research Institute Tasmania Australia

Reviewed by:

Yiorgos Apidianakis, University of Cyprus, Cyprus

Rebecca Leigh Schmidt,

Upper lowa University, USA

*Correspondence:

Miguel Moreno-García miguelmoga2000@yahoo.com.mx; Humberto Lanz-Mendoza

humberto@insp.mx

Specialty section:

This article was submitted to

Microbial Immunology,

a section of the journal

Frontiers in Microbiology

Received: 20 November 2015 Accepted: 06 June 2016

Published: 22 June 2016

Citation:

Vargas V, Moreno-García M, Duarte-Elguea $E$ and Lanz-Mendoza H (2016) Limited Specificity in the Injury and Infection Priming against Bacteria in Aedes aegypti Mosquitoes.

Front. Microbiol. 7:975

doi: 10.3389/fmicb.2016.00975 previous infection of Aedes aegypti would enhance survival and/or lead to greater and specific AMP expression after a second exposure to the same or a distinct bacterium. Mosquitoes previously immunized with a low dose of Escherichia coli, but not Staphylococcus aureus, showed increased survival. Although the host protection herein demonstrated was not specific, each bacterium elicited differential AMP expression. These results can be explained by the susceptible-primed-infected (SPI) epidemiological model, which poses that in the evolution of memory-like responses (priming), a pivotal role is played by pathogen virulence, associated host damage, and the host capacity of pathogen recognition.

Keywords: injury and infection priming, insect, antimicrobial peptides, susceptible-primed-infected (SPI), epidemiological model

\section{INTRODUCTION}

Injury and infection priming is a memory-like response that is reportedly elicited in several (but not all) insect groups by bacteria, fungi, viruses, parasites and even inert molecules (LPS, heat-killed bacteria) (Moret and Siva-Jothy, 2003; Sadd et al., 2005; Moret, 2006; Sadd and Schmid-Hempel, 2007; Roth et al., 2009; Hernández-Martínez et al., 2010; Tidbury et al., 2011; Tate and Rudolf, 2012; Christofi and Apidianakis, 2013). Despite the evidence of a widespread phenomenon, the mechanism(s) and molecule(s) associated with the induction of priming are as yet unclear (but see Contreras-Garduño et al., 2015; Ramirez et al., 2015).

There are studies showing that after previous exposure to a pathogen, there was specific protection against a subsequent lethal challenge. For instance, after previous contact of Drosophila melanogaster with different bacteria, individuals were able to increase its survival rate and

Abbreviations: AMP, antimicrobial peptide; CFU, colony forms units; DSCAM, down syndrome cell adhesion molecule; IMD, immune deficiency; JAK/STAT, janus kinase/signal transducers and activators of transcription; LD, lethal doses; PRR, pattern recognition receptor; RPMI, Roswell Park Memorial Institute; SPI, susceptible-primed-infected. 
specific phagocytic activity against the corresponding pathogen (Pham et al., 2007; Christofi and Apidianakis, 2013). While different strains of Pseudomona aeruginosa elicit defense-specific genes (Apidianakis et al., 2005). Anopheles gambiae showed dissimilar responses to Plasmodium berghei and P. falciparum (Rodrigues et al., 2010). However, these studies do not necessarily reveal the specific recognition of a pathogen, but highlights a specificity response degree against different bacteria during priming.

The specificity of the response against infectious agents can promote an expedited immune activation leading to elimination or control of the invader. The energetic cost of a specific response could be lower than that of a generic immune response or one that is continuously activated (Pham and Schneider, 2008). Consequently, the resources spared by the use of a specific immune response can be used for the expression of other traits linked with survival and reproduction.

There has been criticism of the reports posing that previous contact with an infectious agent can afford an insect with improved immunity against a second exposure to the same pathogen. The specificity of the insect memory-like immune response is rejected by some researchers (see Hauton and Smith, 2007) because of the lack of antibodies as well as clonal expansion and differentiation. Nonetheless, the immune system of insects comprises a number of humoral (soluble and membrane-associated) recognition receptors PRRs (Hoffmann and Reichhart, 2002; Strand, 2008). Moreover, the DSCAM is a PRR that can take thousands of different forms through alternative splicing (Watson et al., 2005; Armitage et al., 2014). In A. gambiae, Dscam (AgDscam) is capable of producing pathogen specific splice form repertoires upon bacteria (Gram+ and Gram-) and Plasmodium infection (Dong et al., 2006). Dscam plays a role mainly as an opsonization molecule with an increased affinity to the infectious organisms (Dong et al., 2006; Smith et al., 2011).

Recognition leads to the activation of three signaling pathways: Toll, IMD and the JAK/STAT (Hoffmann and Reichhart, 2002). In Drosophila, these pathways can induce a differential expression of AMPs following a bacterial challenge (Lemaitre et al., 1997; Apidianakis et al., 2005). The differential recognition of pathogens and production of effectors indicates a certain degree of specificity of the immune system.

Puzzlingly, not all insects seem to show this differential activation and regulation of immune pathways. For example, in Aedes aegypti cellular and melanization responses are independent of the bacterial Gram type (see Hillyer et al., 2004). Even more, some AMPs depend on two pathways (Levashina et al., 1998). At first glance, it could seem unlikely that selective AMP production provides an explanation for the subsequent specific protection in some insects. After all, when dipterans are re-infected, their immunity is mediated by granulocytes (Pham et al., 2007; Rodrigues et al., 2010). This through prohemocyte differentiation that increased the granulocyte population and trigger changes in the morphology and binding properties of these cells (Rodrigues et al., 2010), thus conferring enhanced immunity to following parasite infections.
However, there are signaling pathways required for hemocytes to differentiate in response to one or more differentiation factors (Rodrigues et al., 2010; Ramirez et al., 2014, 2015). Immune pathways of hemocytes are activated after pathogen recognition or humoral stimulation, which leads to the production and release of signaling and/or effector molecules, including AMPs (Bartholomay et al., 2004a). Hemocytes can also induce AMP expression in other tissues, such as fat body (a tissue with great AMP production) (Imler and Bulet, 2005). Consequently, it is plausible that increased cellular activity upon reinfection could have an effect on AMP production. In this sense, ContrerasGarduño et al. (2015) compared two groups of A. albimanus in contact with $P$. berghei. One had had prior contact with this pathogen and the other had not. There was greater AMP transcription in the previously infected mosquitoes. Therefore, it is probable that injury and infection priming entails specific cellular and humoral products, possibly interconnected with each other.

Previous studies have reported injury and infection priming in A. aegypti mosquito (Moreira et al., 2009; Bian et al., 2010; Moreno-García et al., 2015), revealing the role of immune pathways and the participation of AMPs in memory-like responses, meaning the ability to enhance or intensify the effectiveness of the immune response after a previous contact with an elicitor of the immune response. However, priming was analyzed by using consecutive challenges with the same pathogen, but without directly testing the specificity of the response. It is important to determine the specificity of the immune response in insects, because the selective pressures exerted by different pathogens could be reflected in their immune strategies.

For this reason, the aim of the present study was to determine whether previous exposure to bacteria enhanced the survival of A. aegypti and/or led to greater AMP expression after a second exposure to the same or a distinct bacteria. For infections, Escherichia coli (Gram-) and Staphylococcus aureus $(\mathrm{Gram}+)$ were used as pathogen models. We documented AMP transcription at three different designated time points after a nonlethal dose (priming induction) and at another three time points following a second exposure 7 days post-priming (Figure 1). With this design, we tried to avoid overlap of the second challenge with the response generated by the priming dose.

The results demonstrate an increased survival of the mosquitoes previously immunized with a low dose of $E$. coli, but not with $S$. aureus. Although this protection was not specific, differential AMP expression proved to be elicited by each bacterium. We discuss these results in light of the SPI epidemiological model developed by Tidbury et al. (2012) and Best et al. (2013). In this model, pathogen virulence, associated host damage, and the host capacity of pathogen recognition have an essential role in the evolution of memory-like responses (priming).

\section{MATERIALS AND METHODS}

Female adult mosquitoes 3-5 days old were used for the experiments. They were reared under insectary conditions 


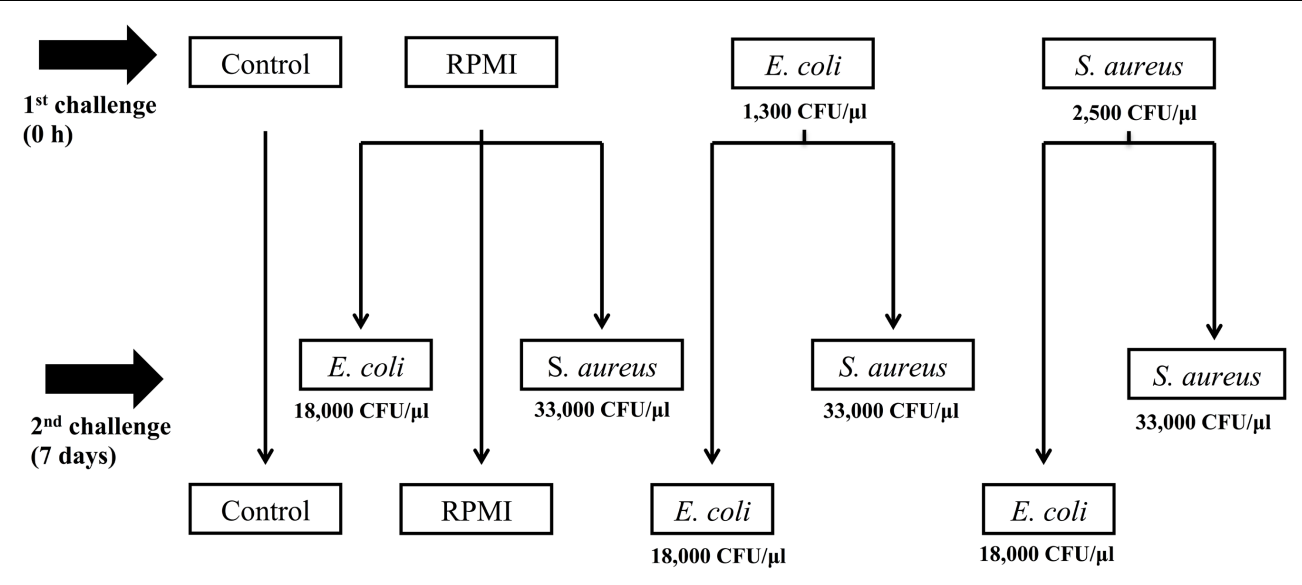

FIGURE 1 | Experimental design of Aedes aegypti immune priming challenge (first injection) and lethal challenge (second injection), using homologous and heterologous challenges with Escherichia coli, Staphylococcus aureus, and RPMI medium only.

(12:12 h light/dark cycle at $\left.25-28^{\circ} \mathrm{C}\right)$ at the Instituto National de Salud Pública (INSP), Mexico. The microbial strains employed for infection were the Gram+ bacterium S. aureus (the $1 \mathrm{MR}$ strain, which is methicillin and oxacillin-resistant, kindly donated by Dr. María Elena Velázquez, INSP) and the Gram- bacterium E. coli (the 01268 strain, which is ampicillin resistant, kindly donated by Dr. Jesús Silva, INSP). To reach the exponential growth phase, the two bacteria were incubated in LB-broth at $37^{\circ} \mathrm{C}$ (200 RPMI), the first for $4 \mathrm{~h}$ and the second for $3.15 \mathrm{~h}$ (for both bacteria final $\mathrm{OD}_{600} \approx 0.350$ ).

\section{Priming and Lethal Doses}

The priming doses $\left(\mathrm{LD}_{0}\right)$ were $2500 \mathrm{CFU} / \mu \mathrm{l}$ for $S$. aureus and $1300 \mathrm{CFU} / \mu \mathrm{l}$ for $E$. coli. For the lethal challenge $\left(\mathrm{LD}_{30}\right)$, we used a higher dose of $E$. coli $\left(18 \times 10^{3} \mathrm{CFU} / \mu \mathrm{l}\right)$ and $S$. aureus $\left(33 \times 10^{3} \mathrm{CFU} / \mu \mathrm{l}\right)$. These doses were previously determined by injecting separate groups of mosquitoes with serial dilutions of each bacterium. We used RPMI (GIBCO-Na $\mathrm{CO}_{3}, 300 \mathrm{mg} / \mathrm{L}$ L-glutamine) as a vehicle for the bacterial injections. This medium was used as a vehicle because mosquito mortality attributable to injection is reduced with this cell culture medium when compared to Schneider and Grace's insect medium (Logrank $\left.x^{2}=131.006, P<0.001\right)$, while no significant difference between PBS, Saline solution $(0.9 \% \mathrm{NaCl})$ and MEM medium $\left(\right.$ Log-rank $\left.x^{2}=1.075-9.18, P>0.05\right)$ (Supplementary Figure S1) was observed. RPMI has been used in other studies (see HerreraOrtíz et al., 2004; Hernández-Martínez et al., 2013a,b; MorenoGarcía et al., 2015).

\section{Injections}

Mosquitoes were cold $\left(4^{\circ} \mathrm{C}\right)$ anesthetized and injected using a pulled glass needle attached to a Drummond Captrol III microinjector. The injection was applied in the abdomen close to the junction of the ventral and dorsal cuticles, volume injected about $\approx 0.1 \mu$ l per mosquito. After injections, mosquitoes were transferred to the insectary and maintained on ad libitum sucrose solution.

\section{Experimental Design}

We first injected two groups of mosquitoes $(n=200)$ with a sublethal dose $\left(\mathrm{LD}_{0}\right)$, using either $S$. aureus or E. coli (Figure 1 ) to trigger injury and infection priming. A third group (the positive control, $n=300$ ) was injected with RPMI only (Figure 1). The negative control group $(n=100)$ was only cold anesthetized for $10 \mathrm{~min}$.

We challenged the mosquitoes with the $\mathrm{LD}_{30}$ dose 7 days after the primary exposure (Figure 1). For this second exposure, half of the $E$. coli primed mosquitoes $(n=100)$ were injected with E. coli (the E. coli-E. coli group) and the other half $(n=100)$ with $S$. aureus (the E. coli-S. aureus group) in order to explore the specificity of the response. Likewise, half of the $S$. aureus primed group $(n=100)$ was given a second injection with $S$. aureus (the $S$. aureus $-S$. aureus group) and the other half $(n=100)$ with E. coli (the S. aureus-E. coli group) in order to explore the specificity of the response. The RPMI injected mosquitoes were divided into three groups for the second injection: the first was administered E. coli (the RPMI-E. coli group, $n=100$ ), the second $S$. aureus (the RPMI-S. aureus group, $n=100$ ), and the third RPMI only (the RPMI-RPMI group, $n=100$; the positive control) (Figure 1). The negative control group (C) was only cold anesthetized again for $10 \mathrm{~min}$.

\section{Survival of Adults after the Lethal Challenge}

After the lethal challenge (second injection), survival was recorded for 29 days. A $\log$-rank $x^{2}$ test was used to detect differences in survival curves between primed (with the same or a different bacterium), unprimed and control groups. The analysis was undertaken using JMP 7.0 (SAS Institute, 2007). Three different biological repetitions were developed.

\section{Antimicrobial Peptide Transcript Dynamics (qRT-PCR Analyses)}

Before the priming-induction injection and at $10 \mathrm{~h}, 24 \mathrm{~h}$, and 7 days post-priming (first injection), ten mosquitoes from each 
group were collected and stored at $-70^{\circ} \mathrm{C}$ to await processing. Likewise, at $10 \mathrm{~h}, 24 \mathrm{~h}$, and 7 days post-lethal challenge (second injection), ten mosquitoes from each group were collected and stored at $-70^{\circ} \mathrm{C}$ until processed.

Total RNA was extracted from each of the groups using $500 \mu \mathrm{l}$ Trizol reagent (Invitrogen) and RNA concentration was measured using Nanodrop. We used $500 \mathrm{ng} / \mu \mathrm{l}$ of the total RNA for cDNA synthesis using the RevertAid Premium Reverse Transcriptase (Thermo Scientific). The resultant cDNA was quantified and welladjusted a $500 \mathrm{ng} / \mu \mathrm{l}$, and $1 \mu \mathrm{l}$ was used for real-time quantitative PCR reactions. The qPCR reaction was performed using genespecific primers for cecropin (CEC Id: AAEL015515-RA, $160 \mathrm{pb}$; forward 5' TCA CAA AGT TAT TTC TCC TGA TCG 3'; reverse 5' GCT TTA GCC CCA GCT ACA AC $3^{\prime}$ ), attacin (ATA Id: AAEL003389-RA, 231 pb; forward 5' TTG GCA GGC ACG GAA TGT CTT G 3'; reverse 5' TGT TGT CGG GAC CGG GAA GTG $3^{\prime}$ ), defensin (DEF Id: AAEL003832-RA, $200 \mathrm{pb}$; forward 5' TTG TTT GCT TCG TTG CTC TTT $3^{\prime}$; reverse $5^{\prime}$ ATC TCC TAC ACC GAA CCC ACT 3') ribosomal protein S7 (internal control, Id: AAEL009496-RA, 190 pb; forward 5' GGG ACA AAT CGG CCA GGC TAT C 3', reverse 5' TCG TGG ACG CTT CTG CTT GTT G $3^{\prime}$ ), and Maxima SYBR Green/ROX qPCR Master Mix (Thermo Scientific) on a StepOne Plus Real-Time PCR system (Applied Biosystem). These sequences were used previously in a priming study with A. aegypti (Moreno-García et al., 2015).

Relative quantification of mRNA levels was done by the $2^{-\Delta \Delta C_{T}}$ method, and primer efficiencies were calculated by measuring how the standard $\Delta C_{\mathrm{T}}$ varied with template serial dilutions (PCR efficiency is about $95-99 \%$ for each AMP). For all trials, the ribosomal protein gene $S 7$ was used as the reference. The levels of $C E C, D E F$, and ATA were normalized with respect to the $\mathrm{S} 7$ transcript of the same sample. Melting curve analyses confirmed that only cDNA, and not genomic DNA, was amplified. The relative expressions of AMP's were represented in ratios of 0 to 1 , because the differences between transcript expressions of AMP were extremely disproportional. Therefore, we homogenize these differences of copy number in ratios for the three AMP genes. Three independent trials were conducted, each analyzed in duplicate.

Differences between groups were analyzed with a one-way ANOVA. Where significant ANOVA differences were found, an LSD-Fisher post hoc was implemented to identify the nature of these differences. Values are presented as the mean \pm SE. Analyses were carried out using Statistica 7.0 (StatSoft, Inc.).

\section{RESULTS}

\section{Survival}

The survival analysis revealed that exposure to a low dose of E. coli delayed significantly mortality of mosquitos against the lethal challenge of the same bacterium. That is, there was a higher survival rate for the E. coli-E. coli than RPMI-E. coli group (Logrank $x^{2}=32.143, P<0.00001$ ) (Figure $2 \mathrm{~A}$ ). The results are from three different biological repetitions. Moreover, priming with $E$. coli also provided protection against a lethal challenge with S. aureus (Figure 2B).
However, previous exposure to $S$. aureus did not elicit priming against the lethal challenge with the same bacterium, evidenced by the fact that the survival of the $S$. aureus-S. aureus mosquitoes did not differ from that of the RPMI-S. aureus group (Logrank $x^{2}=1.014, P=0.602$ ) (Figure 2C). Furthermore, previous exposure to $S$. aureus did not elicit a protective response against the lethal challenge with $E$. coli $\left(\right.$ Log-rank $\left.x^{2}=3.718, P=0.293\right)$ (Figure 2D). Regarding the lethal challenge, E. coli showed a greater negative impact on the survival of mosquitoes than $S$. aureus, suggesting that impact of the former probably was continuous through time.

\section{Dynamics of Antimicrobial Peptide Transcripts (Response Induced by E. coli Priming)}

Before the first injection, no differences were observed in the relative mRNA levels of transcripts for any of the three peptides (cecropin, attacin or defensin, Figure 3). Ten hours after the first injection using RPMI or E. coli, a slight cecropin relative expression was observed in both cases. Attacin and defensin expression was higher after the first injection using RPMI or E. coli, although there were no differences between these two injected groups (RPMI vs. E. coli, Figure 3A). At 24 h postinjection, a decreased expression of the transcripts was observed for both groups, and at the 7th day post-injection values were close to 0.1 .

Compared to the value found after the first injection, $10 \mathrm{~h}$ after the lethal challenge (second injection) with E. coli (in the E. coli-E. coli and RPMI-E. coli groups), there was an increased expression of cecropin and defensin (one and fourfold, respectively), with no differences between these two groups (Figure 3A). Attacin also showed an increased expression, which was higher for the RPMI-E. coli than the E. coli-E. coli group (LSD Post hoc, $P=0.002$; Figure 3A; Supplementary Table S1). For the RPMI-RPMI group, the AMP expression was lower than that found in the other injected groups (Figure 3A). The increased AMP expression after the second injection can be attributed to the higher $E$. coli dose used for the lethal challenge than for priming. At $24 \mathrm{~h}$ post-lethal challenge (second injection), we observed a priming effect with regard to attacin and defensin, evidenced by the higher level of these peptides in the E. coli$E$. coli than RPMI-E. coli group (LSD post hoc, $P=0.002$ and $P=0.004$, respectively; Figure 3A). Cecropin showed an increased expression in both these groups, but there was no difference between the E. coli-E. coli and RPMI-E. coli groups. For the RPMI-RPMI group, the AMP expression remained at a low level compared to the other injected groups. At the 7 th day post-lethal challenge, the AMP expression in all groups declined to around 0.2 or 0.1 , with no differences detected between groups.

At $10 \mathrm{~h}, 24 \mathrm{~h}$, and 7 days post-lethal challenge, there were no significant differences in cecropin and defensin expression between the E. coli-E. coli and E. coli-S. aureus groups (Figure 3B). However, at $24 \mathrm{~h}$ post-lethal challenge, a lower expression of attacin was observed in the E. coli-S. aureus than E. coli-E. coli group (see Figure 3B; Supplementary Table S1). These results suggest a dissimilar AMP expression induced by 

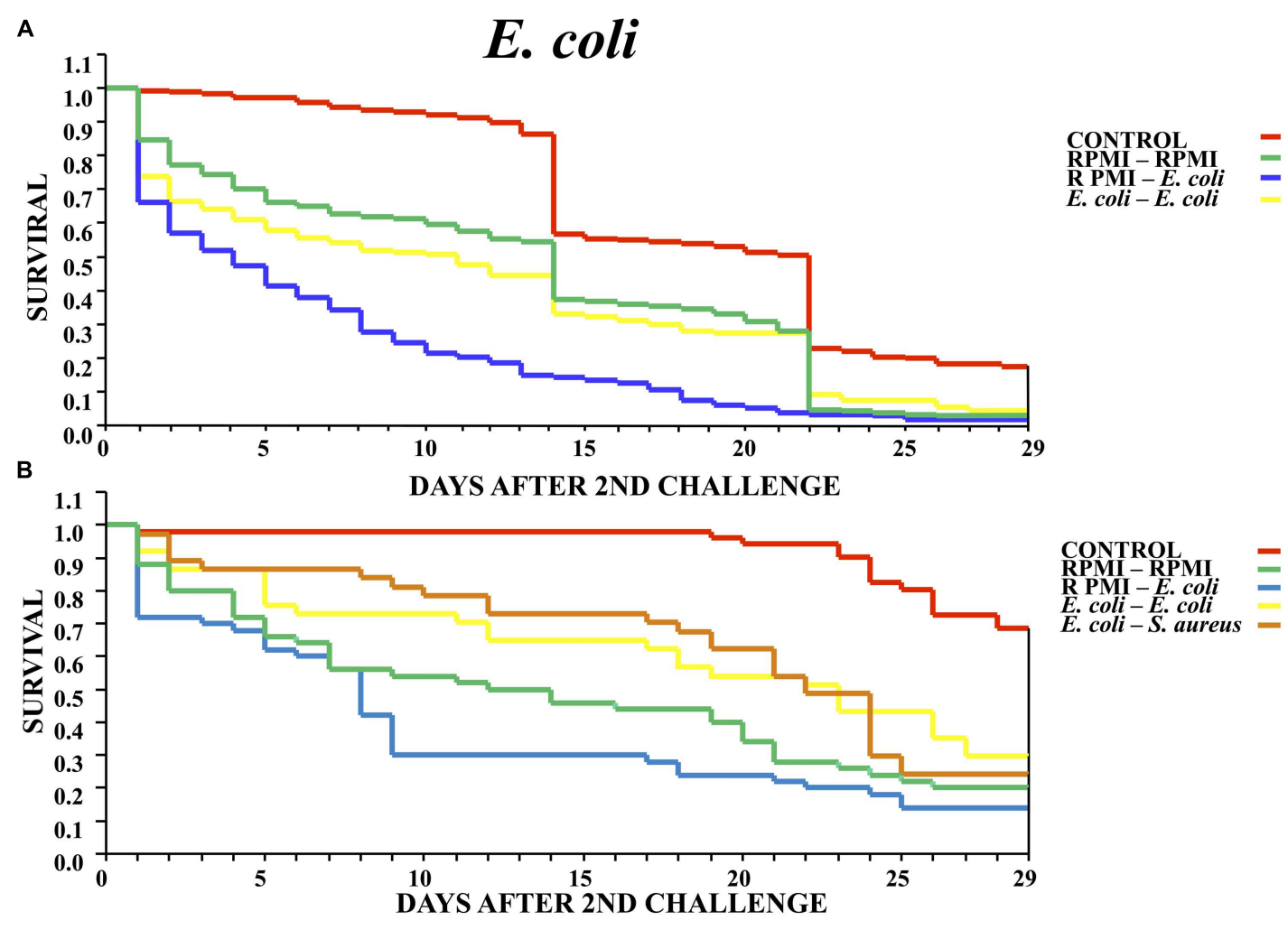

C
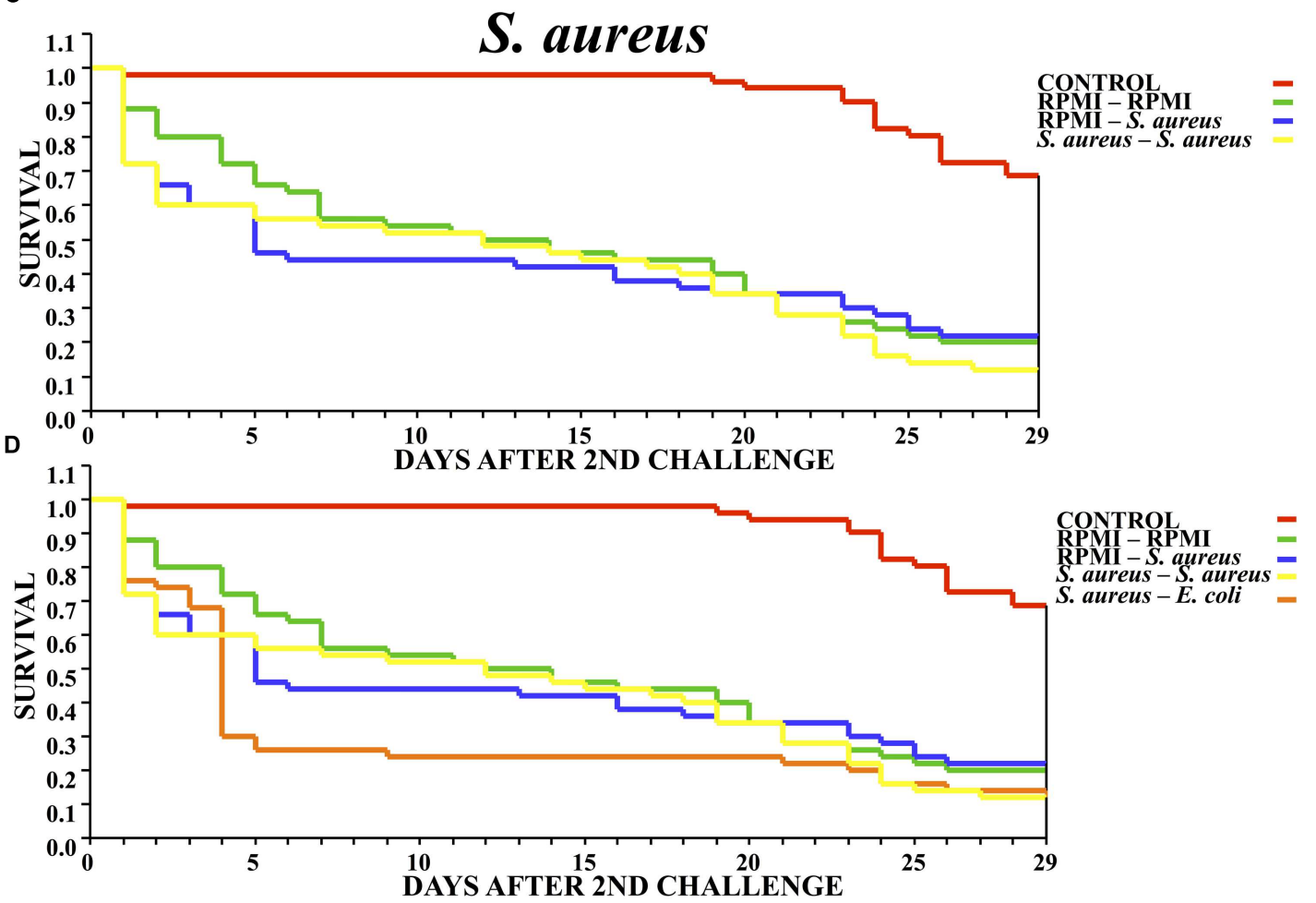

FIGURE 2 | Survival curves for mosquitoes after the second challenge, showing the results when using homologous and heterologous challenges with E. coli, S. aureus and RPMI medium only. (A) Second injection with E. coli or RPMI medium only, after first injection with E. coli or RPMI medium only. (B) Second injection with E. coli, S. aureus, or RPMI medium only, after first injection with E. coli or RPMI medium only. (C) Second injection with S. aureus or RPMI medium only, after first injection with S. aureus or RPMI medium only. (D) Second injection with S. aureus, E. coli, or RPMl medium only, after first injection with S. aureus or RPMI medium only. Control were mosquitoes that were not treated (cold-only). Data are expressed as the mean \pm SE from 3 independent experiments. 


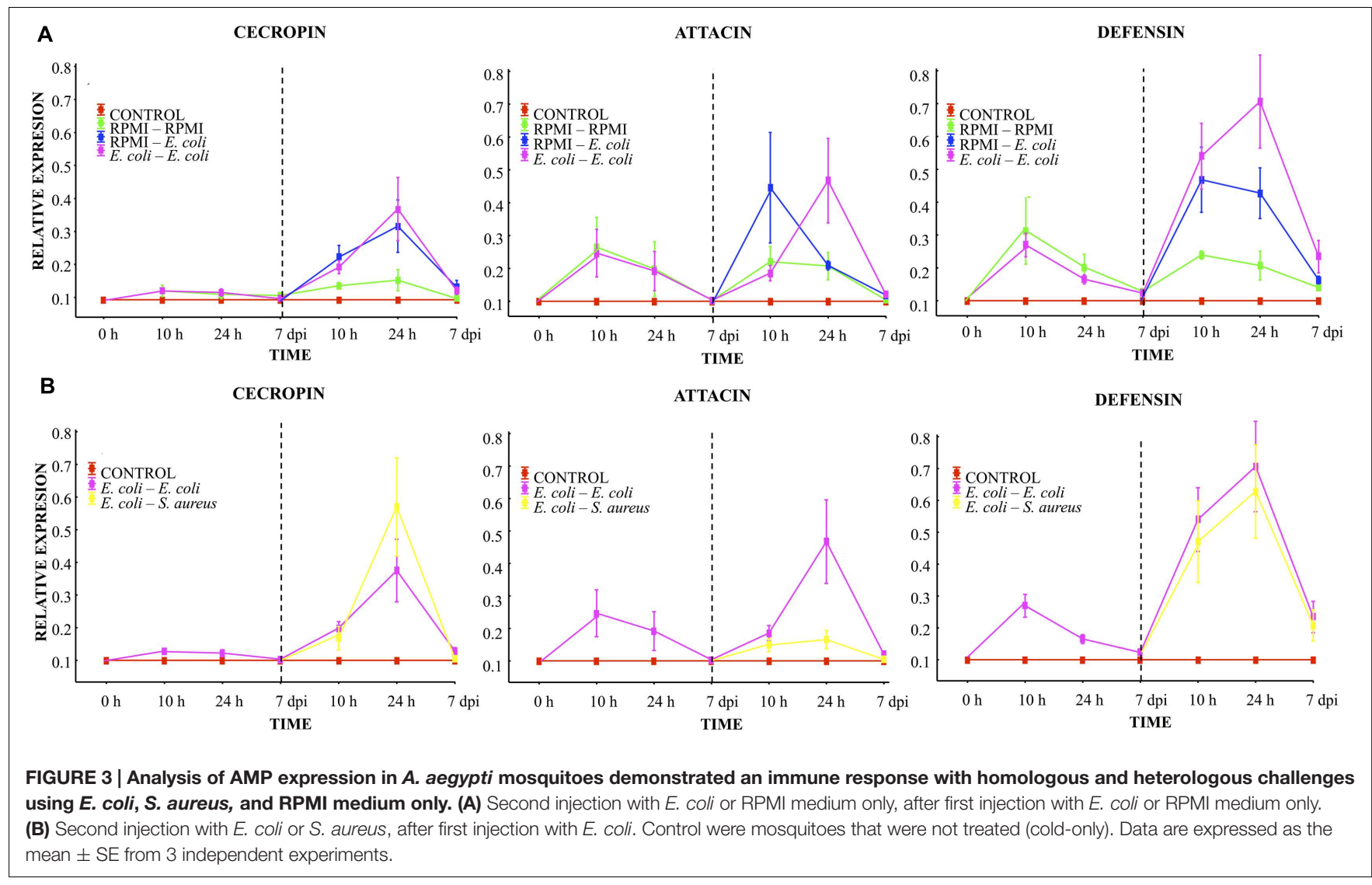

each bacterium. Defensin and cecropin expression could be correlated with the observed enhanced survival found in both of these groups, while attacin expression only correlated with the survival of the E. coli-E. coli group.

\section{Dynamics of the Antimicrobial Peptide Transcripts (Response Induced by S. aureus Priming)}

At $10 \mathrm{~h}, 24 \mathrm{~h}$, and 7 days post-injection with the low dose of S. aureus (first injection), low AMP expression was observed. Attacin and defensin expression was higher than that of cecropin. However, no differences were found between the RPMI and $S$. aureus groups (Figure 4A). A decreased expression of the transcripts was observed in all groups at $24 \mathrm{~h}$ post-injection, reaching values close to 0.1 by the 7 th day.

Compared to the expression after the first injection, $10 \mathrm{~h}$ after the lethal challenge a significant increase in defensin and a slight increment of cecropin were observed in both the $S$. aureus-S. aureus and RPMI-S. aureus groups, with no differences between groups (Figure 4A). We observed a priming effect at $24 \mathrm{~h}$ postlethal challenge, evidenced by the higher levels of cecropin and defensin found in the $S$. aureus $-S$. aureus than RPMI-S. aureus group (LSD post hoc, $P=0.001$ and $P=0.008$, respectively; Figure 4A, Supplementary Table $S 1$ ). No increased expression of attacin was detected at any time point for the RPMI-S. aureus, S. aureus-S. aureus, or E. coli-S. aureus group.
At $10 \mathrm{~h}$ post-lethal challenge, there was significantly greater cecropin and defensin expression for the S. aureus-E. coli than S. aureus-S. aureus group (Figure 4B; Supplementary Table S1). Likewise, $24 \mathrm{~h}$ after the lethal challenge the attacin expression was greater for the $S$. aureus-E. coli than $S$. aureus-S. aureus group (Figure 4B; Supplementary Table S1).

Injection damage (represented by the RPMI-RPMI group, Supplementary Table S2) triggered AMP expression. This expression showed the same trend after the first and second injection (Figure 4A). However, levels of expression after the second injection were below those of the first injection for all groups. This result and the low attacin expression in the E. coli-S. aureus, S. aureus-S. aureus, and RPMI-S. aureus groups suggest that $S$. aureus was unable to induce attacin expression.

\section{DISCUSSION}

The present study demonstrates that in females of $A$. aegypti, priming with a low dose of $E$. coli elicited a protective response against a subsequent lethal challenge with the same bacteria or with $S$. aureus. This protective response correlated with the expression of attacin and defensin.

Contrarily, exposure to a low dose of $S$. aureus did not improve the survival of mosquitoes after a lethal challenge with $S$. aureus or E. coli. However, there was an increased expression of defensin and cecropin in the group administered a low 


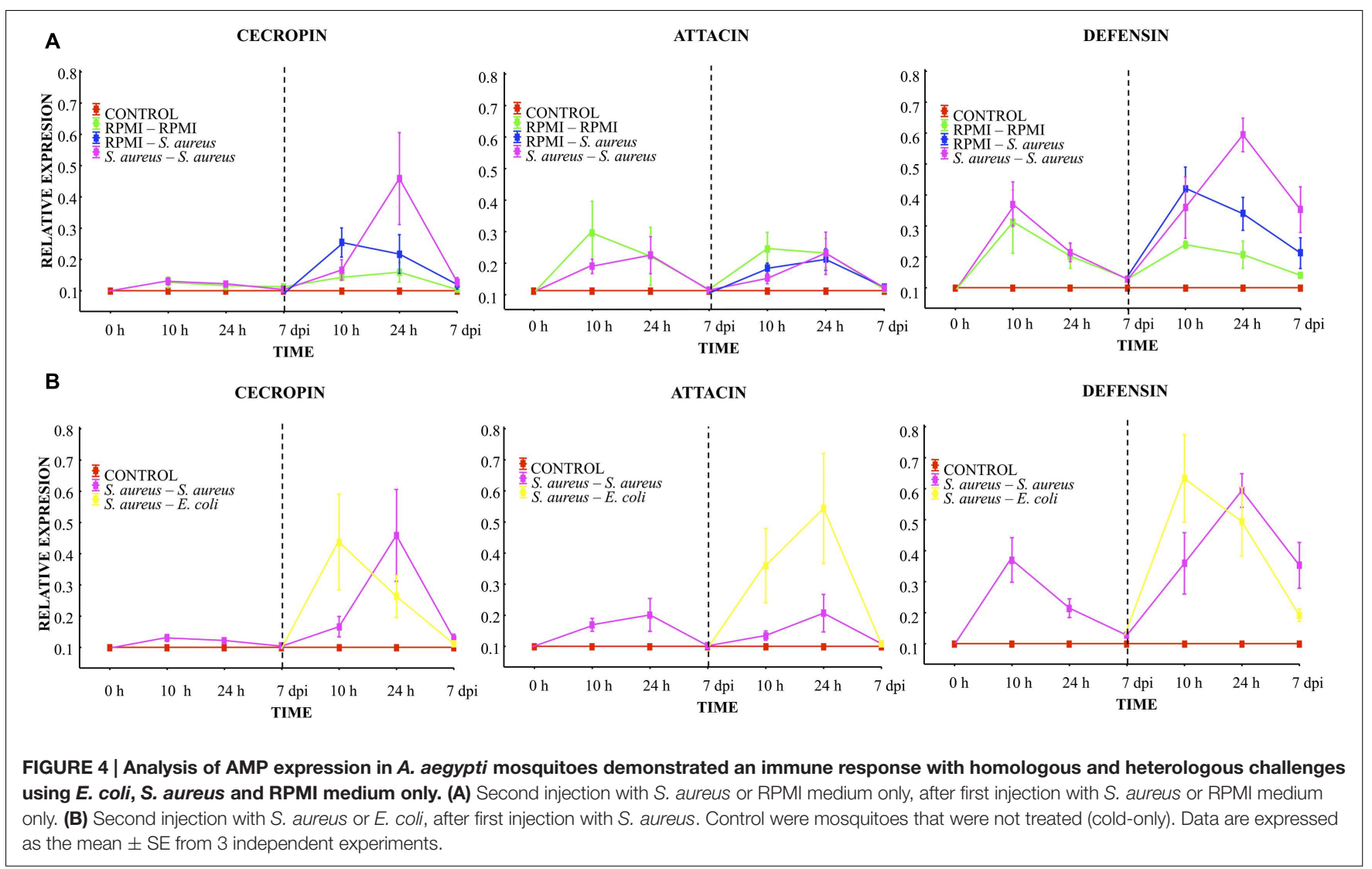

dose of $S$. aureus followed by a lethal challenge with the same bacterium. Additionally, there was an increased expression not only of defensin and cecropin but also of attacin in heterologouschallenged group (S. aureus-E coli), indicating that the two bacteria elicited a differential AMP expression. These results suggest a quasi-specificity (sensu Rowley and Powell, 2007) in AMP expression induced by the lethal challenge.

Although it has been demonstrated that some invertebrates express injury and infection priming (for one or more pathogens), and in some cases specificity of the immune response, evidence in other insect groups reveals that a memory-like response apparently does not exist (e.g., Pham et al., 2007; González-Tokman et al., 2010; Reber and Chapuisat, 2012; Dubuffet et al., 2015; Wu et al., 2015). Our results show that $S$. aureus induced priming in terms of the expression level of AMPs, but not in regard to survival. To our knowledge, this is the first report demonstrating this effect.

The non-ubiquitous expression of priming (specific or otherwise) in insects has been explained by the concept of the inducible costs of priming (Moret and Schmid-Hempel, 2000; González-Tokman et al., 2010). Likewise, the short life span of some insect groups limits the chance of a secondary encounter with the same pathogen (Little and Kraaijeveld, 2004). The SPI model proposes that pathogens producing damage select for greater priming to reduce the negative effects of the infection. But this also depends on the level of protection afforded against future exposure (Tidbury et al., 2012; Best et al., 2013).
In Galleria mellonella, (Mowlds et al., 2010) the authors demonstrated the existence of a threshold below which the immune response is aimed at solely containing the infection. Above this threshold, the immune response not only contains the infection but also protects the host from subsequent contact with the same pathogen. Hence, the host is capable of optimally tuning its response by sensing the infection levels. Our results show that after the lethal challenge, E. coli had greater impact than $S$. aureus (observed by the higher mortality of the RPMIE. coli and S. aureus-E. coli groups), implying a greater chance of damage by this gram- bacteria. Contrarily, the $S$. aureusS. aureus, RPMI-S. aureus, and RPMI-RPMI groups showed similar mortality curves (Figure 2C). In accordance with the SPI injury and infection priming theory, E. coli induced memorylike responses to prevent or diminish damage, leading to greater survival of mosquitoes after injection with a lethal dose.

Compared to E. coli, S. aureus apparently produced lower damage levels to the mosquitoes. Despite the increased cecropin and defensin levels in the $S$. aureus-S. aureus group after the second injection, and the effective activity of these AMPs against Gram-positive bacteria (Lowenberger et al., 1995, 1999; Bartholomay et al., 2004b; Bulet and Stöcklin, 2005), their expression was not sufficient to enhance the survival of primed mosquitoes. Since $S$. aureus had a low effect on the integrity of vital host processes, if mosquitoes were infected with a higher dose they would likely not sense any notable increase in damage, meaning that the organism should not make any 
increased investment in immune response without a survival compensation. The activation of the immune transcriptional pathways may be influenced by several factors, including damage, recognition of the microorganism responsible for producing the damage, and a recovery threshold (van Baalen, 1998; Moreno-García et al., 2014a). After the lethal challenge, the increased expression of defensin activated by $S$. aureus was probably related to the degree of damage to the mosquito, but not to a real compensation in terms of survival. Our results also showed that injury itself (RPMI-RPMI group) could have an impact on the survival, but not in AMPs expression. We hypothesize that mosquito cannot control damage without pathogen sensing. The immune response is turned off when pathogen and damage reach a threshold level where negative effects are "insignificant" and when no more danger molecules (self or non-self) are produced (Chambers and Schneider, 2012). Probably, the damage, without the bacteria, produces an impairment of the regulation of the immune response (ROS, PO but not AMP as figures showed) decreasing mosquitoes survival. Endogenous damage-associated molecular patterns (DAMPs) or danger signals associated with tissue damage can induce innate trained immunity through epigenetic regulation of transcriptional programs (Netea et al., 2011). According to the danger model, damage to tissues induce the release of DAMPs, allowing the proper type and level intensity of the immune response against a certain pathogen (Crisşan et al., 2016).

This trained immunity induced by previously encountered pathogens may also help to respond rapidly and appropriately to the next challenge. However, it seems that in A. aegypti the concurrence of damage and the pathogen leaded to an optimal immune response. Several DAMPs have been found in insects (Moreno-García et al., 2014b). It will be very interesting to determine the DAMPs particularly involved in the A. aegypti's priming against bacteria.

Pathogen presence over several generations could be also required to generate a proper recognition and therefore an investment in priming (Best et al., 2013). In the wild, the microbiota of $A$. aegypti is mostly constituted by Gram-negative bacteria (Zouache et al., 2011; Ramirez et al., 2012). Therefore, it is possible that the mosquito is prone to a priming response when interacting with $E$. coli or other Gram-negative bacteria, while investment in priming and enhanced immunity could be quite costly in relation to the interaction between the mosquito and an uncommon Gram positive bacterium (such as $S$. aureus). The non-expression of attacin in the interaction between the mosquito and $S$. aureus could be related to the specificity of immunity, and particularly to the cost of production of attacin in response or to an infrequent gram + bacterium ( $S$. aureus). However, this explanation needs further research.

\section{REFERENCES}

Apidianakis, Y., Mindrinos, M. N., Xiao, W., Lau, G. W., Baldini, R. L., Davis, R. W., et al. (2005). Profiling early infection responses: Pseudomonas aeruginosa eludes host defenses by suppressing antimicrobial peptide gene expression.
In summary, we have provided evidence for a quasispecific injury and infection priming in $A$. aegypti at the AMP transcriptional level. However, molecular priming in this study did not always correlate with increased mosquito survival. It is likely that investment in the immune response depends on several factors, including pathogen virulence, level of infection, host damage, pathogen presence over several generations, and frequency of contact with the pathogen in the microenvironment of an insect. The mechanisms of immune response in insects include reactions and molecules that are interconnected. Therefore, we cannot exclude the possibility that the memory-like response also depended on cellular activity and/or other humoral molecules not measured herein. The present results suggest that not all the immune effectors may undergo a simultaneous increase in response to pathogens. Priming against bacteria seems to rely on the molecules released after damage (DAMPs) caused by infection, the level of protection afforded against future infections, and probably the prevalence of a pathogen across generations.

\section{AUTHOR CONTRIBUTIONS}

Conceived and designed the experiments: MM-G, VV, HL-M. Performed the experiments: MM-G, VV, ED-E. Analyzed the data: MM-G, VV. Contributed reagents/materials/analysis tools: HL-M. Wrote the paper: MM-G, VV, HL-M.

\section{ACKNOWLEDGMENTS}

We thank María Guadalupe Hernández-Martínez, Inci RamírezBello, and Nalu Davila-Delgado for helping to raise and inject mosquitoes. We thank Alfredo Lagunas-Martínez for the qRTPCR advice. We thank Bruce Allan Larsen for proofreading this paper for the use of English. V. Vargas thanks CONACYT for a Master fellowship (399721/269766) of Posgrado de Ciencias Biológicas, Universidad Nacional Autónoma de México and Centro de Investigaciones Sobre Enfermedades Infecciosas, Instituto Nacional de Salud Pública. MM-G. thanks CONACYT for a postdoctoral fellowship (PNPC, SEP-CONACYT, 290662 INSP) and a grant from Fondo Sectorial de Investigación para la Educación, SEP-CONACYT, Proyect \# 183027.

\section{SUPPLEMENTARY MATERIAL}

The Supplementary Material for this article can be found online at: http://journal.frontiersin.org/article/10.3389/fmicb. 2016.00975

Proc. Natl. Acad. Sci. U.S.A. 102, 2573-2578. doi: 10.1073/pnas.04095 88102

Armitage, S. A. O., Peuß, R., and Kurtz, J. (2014). Dscam and pancrustacean immune memory - a review of the evidence. Dev. Comp. Immunol. 48, 315-323. doi: 10.1016/j.dci.2014.03.004 
Bartholomay, L. C., Cho, W. L., Rocheleau, T. A., Boyle, J. P., Beck, E. T., Fuchs, J. F., et al. (2004a). Description of the transcriptomes of immune response-activated hemocytes from the mosquito vectors Aedes aegypti and Armigeres subalbatus. Infect. Immun. 72, 4114-4126. doi: 10.1128/IAI.72.7.4114-4126.2004

Bartholomay, L. C., Fuchs, J. F., Cheng, L. L., Beck, E. T., Vizioli, J., Lowenberger, C., et al. (2004b). Reassessing the role of defensin in the innate immune response of the mosquito, Aedes aegypti. Insect Mol. Biol. 13, 125-132. doi: 10.1111/j.0962-1075.2004.00467.x

Best, A., Tidbury, H., White, A., and Boots, M. (2013). The evolutionary dynamics of within-generation immune priming in invertebrate hosts. J. R. Soc. Interface 10:20120887. doi: $10.1098 /$ rsif.2012.0887

Bian, G., Xu, Y., Lu, P., Xie, Y., and Xi, Z. (2010). The endosymbiotic bacterium Wolbachia induces resistance to dengue virus in Aedes aegypti. PLoS Pathog. 6:e1000833. doi: 10.1371/journal.ppat.1000833

Bulet, P., and Stöcklin, R. (2005). Insect antimicrobial peptides: structures, properties and gene regulation. Protein Pept. Lett. 12, 3-11. doi: $10.2174 / 0929866053406011$

Chambers, M. C., and Schneider, D. S. (2012). Pioneering immunology: insect style. Curr. Opin. Immunol. 24, 10-14. doi: 10.1016/j.coi.2011.11.003

Christofi, T., and Apidianakis, Y. (2013). Drosophila immune priming against Pseudomonas aeruginosa is short-lasting and depends on cellular and humoral immunity. F1000Res. 2:6. doi: 10.12688/f1000research.2-76.v1

Contreras-Garduño, J., Rodríguez, M. C., Martínez, S. H., Barnetche, J. M., Alvarado, A., Izquierdo, J., et al. (2015). Plasmodium berghei induced priming in Anopheles albimanus independently of bacterial co-infection. Dev. Comp. Immunol. 52, 172-181. doi: 10.1016/j.dci.2015.05.004

Crisşan, T., Netea, M. G., and Joosten, L. A. B. (2016). Innate immune memory: implications for host responses to damage-associated molecular patterns. Eur. J. Immunol. 4, 817-828. doi: 10.1002/eji.201545497

Dong, Y., Taylor, H. E., and Dimopoulos, G. (2006). AgDscam, a hypervariable immunoglobulin domain-containing receptor of the Anopheles gambiae innate immune system. PLoS Biol. 4:e229. doi: 10.1371/journal.pbio.0040229

Dubuffet, A., Zanchi, C., Boutet, G., Moreau, J., Teixeira, M., and Moret, Y. (2015). Trans-generational immune priming protects the eggs only against gram-positive bacteria in the mealworm beetle. PLoS Pathog. 11:e1005178. doi: 10.1371/journal.ppat.1005178

González-Tokman, D. M., González-Santoyo, I., Lanz-Mendoza, H., and Córdoba Aguilar, A. (2010). Territorial damselflies do not show immunological priming in the wild. Physiol. Entomol. 35, 364-372. doi: 10.1111/j.13653032.2010.00752.x

Hauton, C., and Smith, V. J. (2007). Adaptive immunity in invertebrates: a straw house without a mechanistic foundation. Bioessays 29, 1138-1146. doi: 10.1002/bies. 20650

Hernández-Martínez, P., Naseri, B., Navarro-Cerrillo, G., Escriche, B., Ferré, J., and Herrero, S. (2010). Increase in midgut microbiota load induces an apparent immune priming and increases tolerance to Bacillus thuringiensis. Environ. Microbiol. 12, 2730-2737. doi: 10.1111/j.1462-2920.2010.02241.x

Hernández-Martínez, S., Barradas-Bautista, D., and Rodríguez, M. H. (2013a). Diferential dna synthesis in Anopheles albimanus tissues induced by immune challenge with different microorganisms. Arch. Insect Biochem. Physiol. 84, 1-14. doi: 10.1002/arch.21108

Hernández-Martínez, S., Lanz-Mendoza, H., Martínez-Barnetche, J., and Rodríguez, M. H. (2013b). Antimicrobial properties of Anopheles albimanus pericardial cells. Cell Tissue Res. 351, 127-137. doi: 10.1007/s00441-012$1505-6$

Herrera-Ortíz, A., Lanz-Mendoza, H., Martínez-Barnetche, J., HernándezMartínez, S., Villarreal-Treviño, H., Aguilar-Marcelino, L., et al. (2004). Plasmodium berghei ookinetes induce nitric oxide production in Anopheles pseudopunctipennis midguts cultured in vitro. Insect Biochem. Mol. Biol. 34, 893-901. doi: 10.1016/j.ibmb.2004.05.007

Hillyer, J. F., Schmidt, S. L., and Christensen, B. M. (2004). The antibacterial innate immune response by the mosquito Aedes aegypti is mediated by hemocytes and independent of Gram type and pathogenicity. Microbes Infect. 6, 448-459. doi: 10.1016/j.micinf.2004.01.005

Hoffmann, J. A., and Reichhart, J. M. (2002). Drosophila innate immunity: an evolutionary perspective. Nat. Immunol. 3, 121-126. doi: 10.1038/ni0202-121
Imler, J. L., and Bulet, P. (2005). "Antimicrobial peptides in Drosophila: structures, activities and gene regulation," in Mechanisms of Epithelial Defense, eds D. Kabelitz and J.-M. Schröder (Basel: Karger), 1-21.

Lemaitre, B., Reichhart, J. M., and Hoffmann, J. (1997). Drosophila host defense: differential induction of antimicrobial peptide genes after infection by various classes of microorganisms. Proc. Natl. Acad. Sci. U.S.A. 94, 14614-14619. doi: 10.1073/pnas.94.26.14614

Levashina, E., Ohresser, S., Lemaitre, B., and Imler, J. L. (1998). Two distinct pathways can control expression of the gene encoding the Drosophila antimicrobial peptide metchnikowin. J. Mol. Biol. 278, 515-527. doi: 10.1006/jmbi.1998.1705

Little, T. J., and Kraaijeveld, R. (2004). Ecological and evolutionary implications of immunological priming ininvertebrates. Trends Ecol. Evol. 19, 58-60. doi: 10.1016/j.tree.2003.11.011

Lowenberger, C., Bulet, P., Charlet, M., Hetru, C., Hodgeman, B., Christensen, B. M., et al. (1995). Insect immunity: isolation of three novel inducible antibacterial defensins from the vector mosquito, Aedes aegypti. Insect Biochem. Mol. Biol. 25, 867-873. doi: 10.1016/0965-1748(95)00043-U

Lowenberger, C. A., Kamal, S., Chiles, J., Paskewitz, S., Bulet, P., Hoffmann, J. A., et al. (1999). Mosquito-Plasmodium interactions in response to immune activation of the vector. Exp. Parasitol. 91, 59-69. doi: 10.1006/expr.1999.4350

Moreira, L. A., Iturbe-Ormaetxe, I., Jeffery, J. A., Lu, G., Pyke, A. T., Hedges, L. M., et al. (2009). A Wolbachia symbiont in Aedes aegypti limits infection with Dengue, Chikungunya, and Plasmodium. Cell 139, 1268-1278. doi: 10.1016/j.cell.2009.11.042

Moreno-García, M., Condé, R., Bello-Bedoy, R., and Lanz-Mendoza, H. (2014a). The damage threshold hypothesis and the immune strategies of insects. Infect. Genet. Evol. J. 24, 25-33. doi: 10.1016/j.meegid.2009.04.004

Moreno-García, M., Récio-Totóro, B., Claudio Piedras, F., and Lanz-Mendoza, H. (2014b). Injury and immune response: applying the danger theory to mosquitoes. Front. Plants Sci. 5:451. doi: 10.3389/fpls.2014.00451

Moreno-García, M., Vargas, V., Ramírez-Bello, I., Hernández-Martínez, G., and Lanz-Mendoza, H. (2015). Bacterial exposure at the larval stage induced sexual immune dimorphism and priming in adult Aedes aegypti Mosquitoes. PLoS ONE 10:e0133240. doi: 10.1371/journal.pone.0133240

Moret, Y. (2006). "Trans-generational immune priming": specific enhancement of the antimicrobial immune response in the mealworm beetle, Tenebrio molitor. Proc. Biol. Sci. 273, 1399-1405. doi: 10.1098/rspb.2006.3465

Moret, Y., and Schmid-Hempel, P. (2000). Survival for immunity: the price of immune system activation for bumblebee workers. Science 290, 1166-1168. doi: 10.1126/science.290.5494.1166

Moret, Y., and Siva-Jothy, M. T. (2003). Adaptive innate immunity? Responsivemode prophylaxis in the mealworm beetle, Tenebrio molitor. Proc. Biol. Sci. 270, 2475-2480. doi: 10.1098/rspb.2003.2511

Mowlds, P., Coates, C., Renwick, J., and Kavanagh, K. (2010). Dose-dependent cellular and humoral responses in Galleria mellonella larvae following $\beta$-glucan inoculation. Microbes Infect. 12, 146-153. doi: 10.1016/j.micinf.2009.11.004

Netea, M. G., Quintin, J., and van der Meer, J. W. M. (2011). Trained immunity: a memory for innate host defense. Cell Host Microbe 9, 335-361. doi: 10.1016/j.chom.2011.04.006

Pham, L. N., Dionne, M. S., Shirasu-Hiza, M., and Schneider, D. S. (2007). A specific primed immune response in Drosophila is dependent on phagocytes. PLoS Pathog. 3:e26. doi: 10.1371/journal.ppat.0030026

Pham, L. N., and Schneider, D. (2008). "Evidence for specificity and memory in the insect innate immune response," in Insect Immunology, ed. N. Beckage (San Diego, CA: Academic Press), 97-127. doi: 10.1016/B978-012373976-6.50007-0

Ramirez, J. L., de Almeida Oliveira, G., Calvo, E., Dalli, J., Colas, R. A., Serhan, C. N., et al. (2015). A mosquito lipoxin/lipocalin complex mediates innate immune priming in Anopheles gambiae. Nat. Commun. 6:7403. doi: $10.1038 /$ ncomms 8403

Ramirez, J. L., Garver, L. S., Brayner, F. A., Alves, L. C., Rodrigues, J., Molina-Cruz, A., et al. (2014). The role of hemocytes in Anopheles gambiae antiplasmodial immunity. J. Innate Immun. 6, 119-128. doi: 10.1159/000353765

Ramirez, J. L., Souza-Neto, J., Cosme, R. T., Rovira, J., Ortiz, A., Pascale, J. M., et al. (2012). Reciprocal tripartite interactions between the Aedes aegypti midgut microbiota, innate immune system and dengue virus influences vector competence. PLoS Negl. Trop. Dis. 6:e1561. doi: 10.1371/journal.pntd.0001561 
Reber, A., and Chapuisat, M. (2012). No evidence for immune priming in ants exposed to a fungal pathogen. PLoS ONE 7:e35372. doi: 10.1371/journal.pone.0035372

Rodrigues, J., Brayner, F. A., Alves, L. C., Dixit, R., and Barillas-Mury, C. (2010). Hemocyte differentiation mediates innate immune memory in Anopheles gambiae mosquitoes. Science 329, 1353-1355. doi: 10.1126/science.1190689

Roth, O., Sadd, B. M., Schmid-Hempel, P., and Kurtz, J. (2009). Strain-specific priming of resistance in the red flour beetle, Tribolium castaneum. Proc. Biol. Sci. 276, 145-151. doi: 10.1098/rspb.2008.1157

Rowley, A. F., and Powell, A. (2007). Invertebrate immune systems specific, quasi-specific, or nonspecific? J. Immunol. 179, 7209-7214. doi: 10.4049/jimmunol.179.11.7209

Sadd, B., and Schmid-Hempel, P. (2007). Facultative but persistent transgenerational immunity via the mother's eggs in bumblebees. Curr. Biol. 17, 1046-1047. doi: 10.1016/j.cub.2007.11.007

Sadd, B. M., Kleinlogel, Y., Schmid-Hempel, R., and Schmid-Hempel, P. (2005). Trans-generational immune priming in a social insect. Biol. Lett. 1, 386-388. doi: $10.1098 / \mathrm{rsbl} .2005 .0369$

Smith, P. H., Mwangi, J. M., Afrane, Y. A., Yan, G., Obbard, D. J., RanfordCartwright, L. C., et al. (2011). Alternative splicing of the Anopheles gambiae Dscam gene in diverse Plasmodium falciparum infections. Malar. J. 10:156. doi: $10.1186 / 1475-2875-10-156$

Strand, M. R. (2008). "Insect hemocytes and their role in immunity," in Insect Immunology, ed. N. E. Beckage (Cambridge, MA: Academic Press), 25-48.

Tate, A. T., and Rudolf, V. H. W. (2012). Impact of life stage specific immune priming on invertebrate disease dynamics. Oikos 121, 1083-1092. doi: 10.1111/j.1600-0706.2011.19725.x

Tidbury, H. J., Best, A., and Boots, M. (2012). The epidemiological consequences of immune priming. Proc. R. Soc. B Biol. Sci. 279, 4505-4512. doi: $10.1098 /$ rspb.2012.1841
Tidbury, H. J., Pedersen, A. B., and Boots, M. (2011). Within and transgenerational immune priming in an insect to a DNA virus. Proc. Biol. Sci. 278, 871-876. doi: 10.1098/rspb.2010.1517

van Baalen, M. (1998). Coevolution of recovery ability and virulence. Proc. Biol. Sci. 265, 317-325. doi: 10.1098/rspb.1998.0298

Watson, F. L., Püttmann-Holgado, R., Thomas, F., Lamar, D. L., Hughes, M., Kondo, M., et al. (2005). Extensive diversity of Ig-superfamily proteins in the immune system of insects. Science 309, 1874-1878. doi: $10.1126 /$ science. 1116887

Wu, G., Yi, Y., Sun, J., Li, M., and Qiu, L. (2015). No evidence for priming response in Galleria mellonella larvae exposed to toxin protein PirA2B2 from Photorhabdus luminescens TT01: an association with the inhibition of the host cellular immunity. Vaccine 33, 6307-6313. doi: 10.1016/j.vaccine.2015.09.046

Zouache, K., Raharimalala, F. N., Raquin, V., Tran-Van, V., Raveloson, L. H. R., Ravelonandro, P., et al. (2011). Bacterial diversity of field-caught mosquitoes, Aedes albopictus and Aedes aegypti, from different geographic regions of Madagascar. FEMS Microbiol. Ecol. 75, 377-389. doi: 10.1111/j.15746941.2010.01012.x

Conflict of Interest Statement: The authors declare that the research was conducted in the absence of any commercial or financial relationships that could be construed as a potential conflict of interest.

Copyright (c) 2016 Vargas, Moreno-García, Duarte-Elguea and Lanz-Mendoza. This is an open-access article distributed under the terms of the Creative Commons Attribution License (CC BY). The use, distribution or reproduction in other forums is permitted, provided the original author(s) or licensor are credited and that the original publication in this journal is cited, in accordance with accepted academic practice. No use, distribution or reproduction is permitted which does not comply with these terms. 